\title{
Management of pleural recurrence after curative resection of thymoma
}

\author{
Marco Lucchi, MD, ${ }^{\text {a }}$ Federico Davini, MD, ${ }^{a}$ Roberta Ricciardi, MD, ${ }^{a}$ Leonardo Duranti, MD, ${ }^{\text {a Laura Boldrini, MD, }}$ \\ Gerardo Palmiero, MD, ${ }^{\mathrm{c}}$ Fulvio Basolo, MD, ${ }^{\mathrm{b}}$ and Alfredo Mussi, MD ${ }^{\mathrm{a}}$
}

\begin{abstract}
Objective: A complete surgical resection is the cornerstone of therapy of thymic tumors. Unfortunately, there is no standard treatment for pleural recurrence. This article describes our overall experience with the surgical treatment of pleural implants in patients who previously underwent resection of a thymoma.
\end{abstract}

\begin{abstract}
Material and Methods: From January 1980 to June 2006, 20 patients previously operated on for a thymoma were operated on for the surgical resection of pleural implants. Patients with the initial Masaoka stage IVA were excluded from our analysis. Our sample comprised 10 male and 10 female patients (12-65 years old). The surgical approach to the resection of the thymoma was as follows: video-assissted thoracic surgery in 2 patients, sternotomy in 13 patients, thoracotomy in 2 patients, and sternothoracotomy in 3 patients. The initial Masaoka stage of the thymoma was IIA in 2 patients, IIB in 7 patients, and III in 11 patients.

Results: The interval between resection of the thymoma and pleural implants ranged from 11 to 156 (median 60) months. Fifteen patients had a thymus-related syndrome (in 13 patients it resulted myasthenia gravis), and in 11 patients it improved or remitted after treatment of the pleural recurrence. All the resections were performed through a posterolateral thoracotomy. Three patients underwent an iterative resection of new pleural implants. At the latest follow-up, 10 patients are still alive ( 8 disease-free) and 10 have died ( 9 of a relapse and 1 of the complications of red cell aplasia). From the pleural recurrence resection, the overall 5- and 10-year survivals are $43.1 \%$ and $25.8 \%$, respectively.

Conclusions: Repeat operation on patients with thymoma pleural recurrences is feasible and safe. It can produce satisfactory results in terms of overall survival and paraneoplastic syndrome control. Moreover, the multimodality treatment could improve the results of surgical treatment.
\end{abstract}

Thymomas are neoplasms arising from the epithelial thymic cells. These tumors rarely metastasize distantly; on the contrary, they can show pleural implants more frequently at diagnosis or during follow-up. ${ }^{1}$ Data in the literature show that pleural implants can also occur several years after the resection of a thymoma, both encapsulated and invasive. ${ }^{2-7}$ Unfortunately, few reports have analyzed the treatment of recurrences of thymoma, ${ }^{3-7}$ and few reports have focused on pleural implants. ${ }^{8-10}$ The treatment of pleural implants is controversial $^{6,8,11-15}$; thus, the aims of our study were (1) to retrospectively analyze our institutional experience on pleural recurrences from thymoma and (2) to discuss the treatment and prognosis of this highly selected group of patients.

\section{PATIENTS AND METHODS}

From January 1980 to June 2006, 20 of 257 patients undergoing resection for a stage I-III thymoma underwent the surgical resection of pleural implants. Patients with initial Masaoka stage IVA were excluded from

From the Division of Thoracic Surgery, Cardiac and Thoracic Department ${ }^{\mathrm{a}}$ and the Division of Pathology, ${ }^{\mathrm{b}}$ University of Pisa, Pisa, Italy, and the Weaning and Pulmonary Rehabilitation Unit, ${ }^{\mathrm{c}}$ Auxilium Vitae, Volterra, Italy.

Received for publication Feb 18, 2008; revisions received Aug 4, 2008; accepted for publication Sept 14, 2008.

Address for reprints: M. Lucchi, MD, Division of Thoracic Surgery, Cardiac and Thoracic Department, University of Pisa, Via Paradisa 2, Pisa 56124, Italy (E-mail: m. lucchi@med.unipi.it).

J Thorac Cardiovasc Surg 2009;137:1185-9

$0022-5223 / \$ 36.00$

Copyright (c) 2009 by The American Association for Thoracic Surgery doi:10.1016/j.jtcvs.2008.09.033 our analysis. Collection of data and follow-up were done by reviewing medical charts and by telephone contacts. Our samples (Table 1) consisted of 10 male and 10 female patients, and the ages of the patients at pleural implant resection ranged from 12 to 65 years old. The surgical approach at the resection of the thymoma was video-assisted thoracic surgery (VATS) in 2 patients (recent patients, both operated on in another hospital), a sternotomy in 13 ( 1 operated on in another institution), a thoracotomy in 2, and a sternothoracotomy in 3 . The initial Masaoka staging of the thymoma was IIA in 2 patients, IIB in 7 patients, and III in 11 patients. Six patients underwent neoadjuvant chemotherapy for an invasive stage III thymoma.

World Health Organization (WHO) histologic types of thymoma were $\mathrm{AB}$ in 1 patient, $\mathrm{B} 1$ in 5 patients, $\mathrm{B} 2$ in 6 patients, and $\mathrm{B} 3$ in 8 patients. Postoperative radiotherapy was offered to all patients of our series (since they all had stage II or III thymomas). No patient underwent adjuvant chemotherapy after resection of the thymoma. No neoadjuvant therapy was performed before the treatment of pleural implants. All patients were approached by a posterolateral thoracotomy; in only 1 patient (previously treated by VATS) was a sternothoracotomy performed. A partial pleurectomy was always performed to resect the implants. On the other hand, a complete pleurectomy/decortication (as for malignant pleural mesothelioma) was never planned. In the case of a wide pleural spread or multiple implants, a small intrapleural catheter was set at the end of the procedure and intrapleural chemotherapy (epidoxorubicin $25 \mathrm{mg} / \mathrm{m}^{2}$ ) was administered on the fifth, seventh, and ninth postoperative day. Since April 2005 (therefore, the last 3 patients), according to our latest in-house protocol, patients underwent hyperthermic intrapleural chemotherapy at $41^{\circ} \mathrm{C}$ to $42^{\circ} \mathrm{C}$ for 1 hour with cisplatin and epidoxorubicin (Figure 1). When the diaphragm was involved, we always performed a partial resection, suturing the break with nonabsorbable single stitches (Figure 2).

We used adjuvant chemotherapy with the scheduling of cisplatinepidoxorubicin-etoposide or carboplatinum-taxol (according to the performance status of the patient) when pleural disease was massive and resection should be considered cytoreductive and not radical. 


\section{Abbreviations and Acronyms \\ VATS $=$ video-assisted thoracic surgery \\ WHO $=$ World Health Organization}

Since November 2000 (last 6 patients) all patients have undergone longterm therapy (6-24 months) with a slow-release octreotide.

The study was approved by our hospital review board.

The statistical analysis was performed by the STATISTICA software (StatSoft, Inc, Tulsa, Okla). Univariable analysis by the Mann-Whitney $U$ test for continuous variables and the $\chi^{2}$ test for discrete variables were used to assess differences of tumor characteristics between patients who had a relapse and those who had not. Survival curves were estimated by the Kaplan-Meier product limit method and were compared by the logrank test.

\section{RESULTS}

The incidence of pleural recurrences in our population of resected thymoma (excluding the stage IVA thymomas and the patients operated on in another hospital) is $6.6 \%$. The median interval between the resection of the thymoma and the pleural implants was 60 months (range 11-156). At the diagnosis of pleural relapse, 15 patients had a thymus-related syndrome (in 13 patients, myasthenia gravis). In all patients it was present at resection of the thymoma, but in 8 patients, after a period of improvement or complete remission, the symptoms worsened. In 9 patients the pleural implants were single whereas in 11 they were multiple. Visceral and parietal pleural disseminated lesions were resected with small margins. Intercostal or pulmonary parenchymal involvement was managed with chest wall resection $(\mathrm{n}=2)$ or wedge resections of the involved lung $(\mathrm{n}=8)$. In 7 patients the pleural implants involved the diaphragm and required a partial resection of the diaphragm.

Our mortality rate was nil. The mean hospital stay was 10.2 days (range $6-22$ days). As regards postoperative morbidity, there was one episode of bleeding (necessitating a reoperation on the second postoperative day), and there were two prolonged air leaks. Three patients underwent iterative resections of pleural implants ( 2 patients underwent a second resection and 1 patient a third). The resection was macroscopically radical in 13 patients and therefore adiuvant chemotherapy was administered only to 7 patients.

With a median follow-up of 98 months (range 13-301), 10 patients are still alive. Eight patients are radiologically disease free, 4 had a local relapse, 5 a systemic relapse, and 2 both a local and systemic relapse. One patient (disease free) died of the complications of red cell aplasia.

The outcomes of the thymus-related syndrome after the treatment of pleural recurrences from thymoma were as follows: 5 patients had a remission, 6 improved, 3 remained in stable condition, and only 1 worsened.

The 10- and 20-year actuarial survivals from the resection of the thymoma were $66 \%$ and $34.3 \%$, respectively. The

TABLE 1. Patient characteristics

\begin{tabular}{lc}
\hline \multicolumn{1}{c}{ Patients's characteristics } & No. \\
\hline Sex (M vs F) & 10 vs 10 \\
Age (y) & 45.8 (range, 12-65) \\
Masaoka staging of the initial thymoma & 2 \\
IIA & 7 \\
IIB & 11 \\
III & \\
Histotype & 1 \\
AB & 5 \\
B1 & 6 \\
B2 & 8 \\
B3 & 60 (range, $11-156)$ \\
Interval (mo) & 15 vs 5 \\
Thymus-related syndrome (yes vs no) & 9 vs 11 \\
No. of implants (single vs multiple) & 7 vs 13 \\
Diaphragmatic implants (yes vs no)
\end{tabular}

5- and 10-year actuarial survivals from the pleural recurrence resection were $43.1 \%$ and $25.8 \%$, respectively (Figure 3). Even though most of the initial thymomas were stage III and B3, neither the Masaoka staging nor the WHO histologic classification significantly affected the survival calculated from the resection of the pleural relapse. The comparison of the survivals according to the disease-free interval ( $<\mathrm{vs}>60$ months) did not reach statistical significance.

Comparing the survival of single versus multiple implants, we found that the difference was statistically significant $(P=.03)$ (Figure 4). Patients with diaphragmatic involvement showed a significantly worse prognosis $(P=$ .01) (Figure 5).

\section{DISCUSSION}

If thymoma is rare, pleural recurrences are even rarer; they account for less than $10 \%$ of resected thymomas. ${ }^{1-5}$

Only a few case reports focused on the surgical treatment of pleural implants that were discovered at follow-up, ${ }^{13}$ whereas most of the articles reported pleural recurrences from thymoma together with mediastinal and systemic relapses. The etiology of the appearance of pleural implants many years after resection of a noninvasive thymoma is not clear. They may have been present at the time of thymoma resection or they may be due to the seeding of tumoral cells during the manipulation of the tumor, especially if the mediastinal pleura has been opened. In our experience, we have always tried to avoid opening the mediastinal pleura in case the thymoma was not invasive. Moreover, we have never performed a thoracoscopy to exclude the presence of pleural disease if the preoperative computed tomographic scan was negative for pleural effusion or implants. On the other hand, we found 2 cases of thymoma (1 stage IIB and 1 stage III) resected by VATS in different hospitals. These patients had a pleural relapse at 38 and 13 months, 

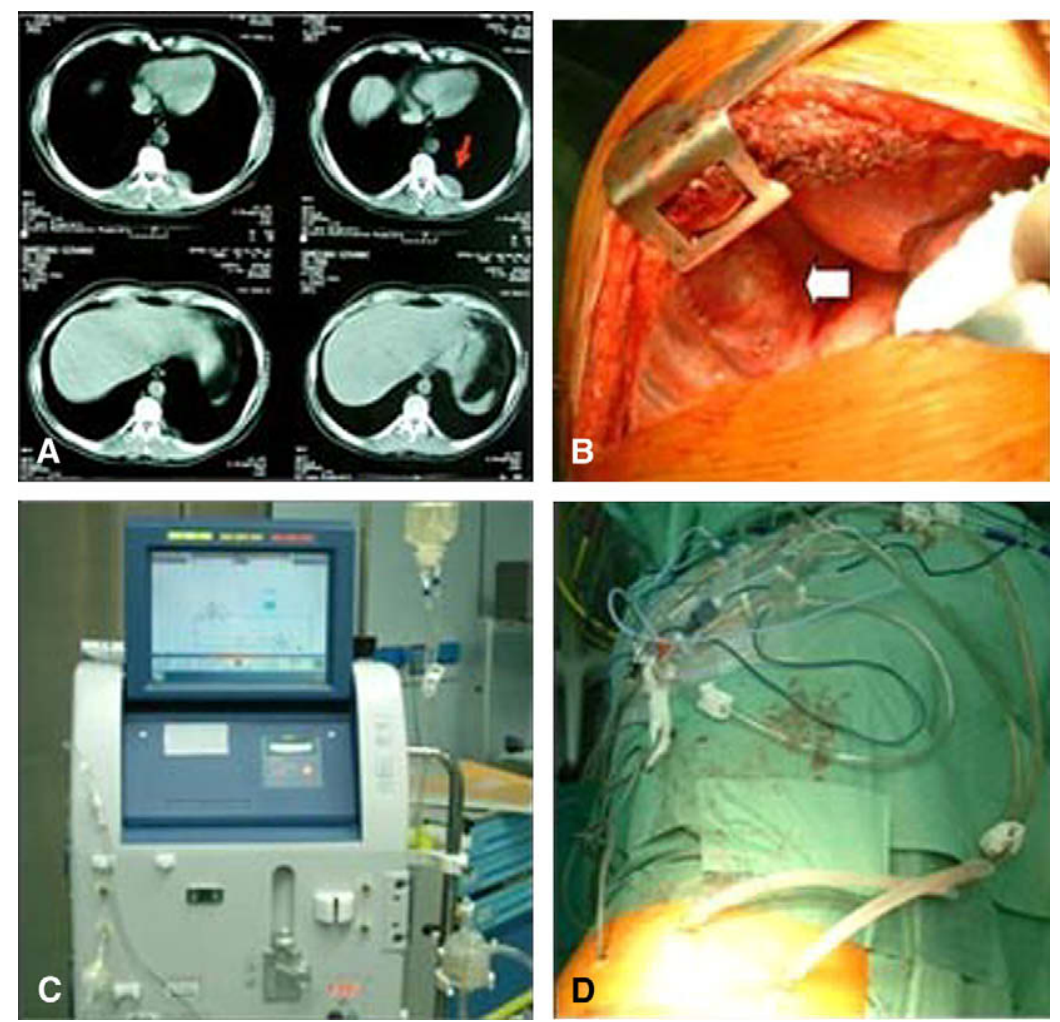

FIGURE 1. A single pleural implant in the left vertebral douche. A, Computed tomography. B, Operative view. After the resection, intrapleural hyperthermic chemotherapy was applied. C, The machine. D, Operative view before starting perfusion.

respectively. This event could stimulate a discussion about the benefit of resecting the thymomas using VATS or robotic technology and the possibility that minimally invasive techniques could expose the patients to a higher risk of pleural relapse.

With regard to the surgical treatment of relapses from thymoma, the articles by Ruffini, ${ }^{3}$ Regnard, ${ }^{5}$ and their associates still remain significant with their overall 30 and

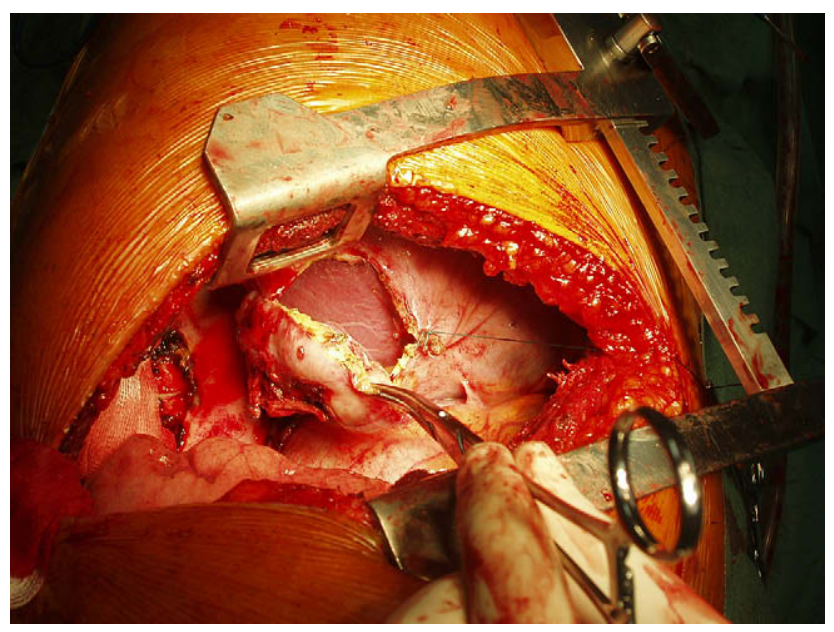

FIGURE 2. Resection of a left diaphragmatic implant.
28 cases, respectively. Both groups of authors concluded that surgical resection is recommended and a poor prognosis can be predicted by the presence of distant recurrences. As a matter of fact, there is no scientific evidence that surgical treatment is superior to other treatments, because in all published studies ${ }^{3,5,6}$ surgery was reserved to resectable recurrent tumors in patients with good clinical conditions; undoubtedly, this is an important clinical selection bias.

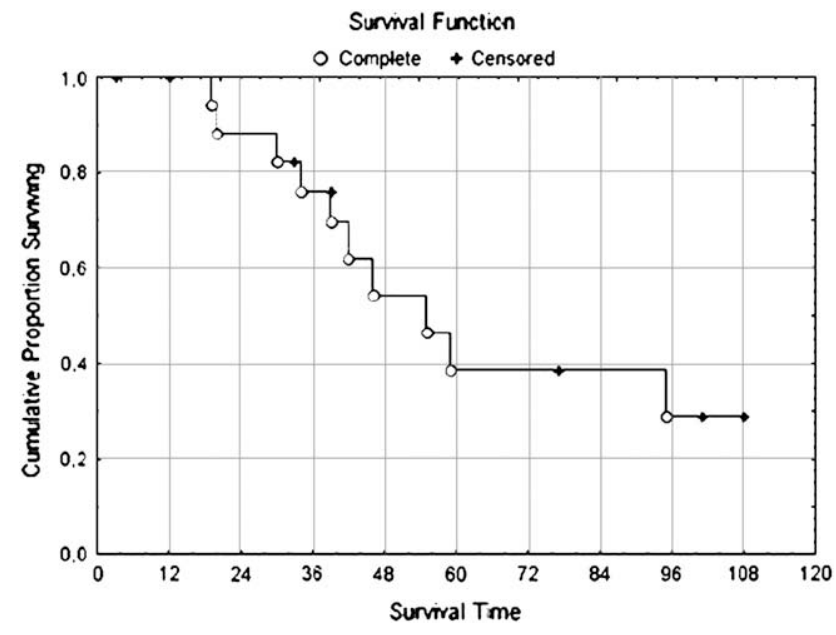

FIGURE 3. Five- and 10-year overall survival calculated from the pleural recurrence resection. 


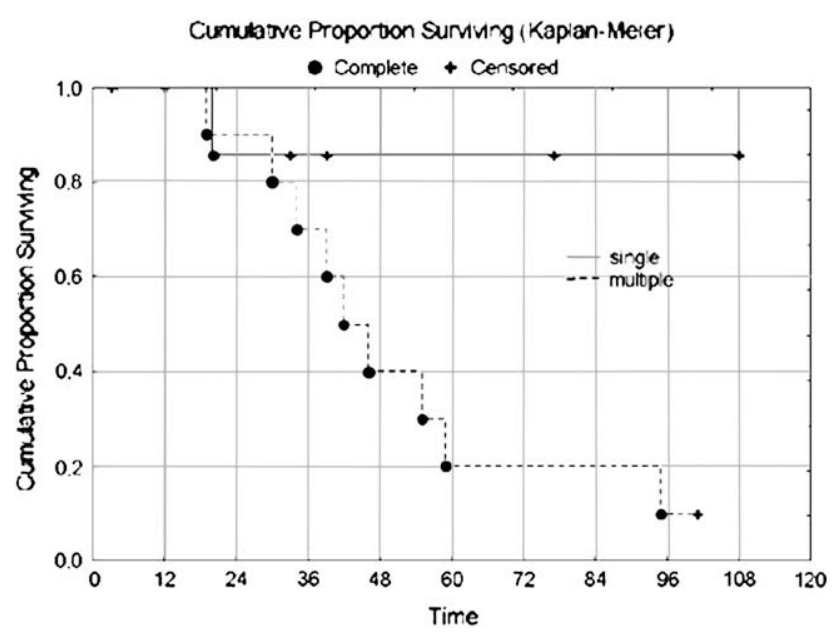

FIGURE 4. Overall survival according to the number of implants (single versus multiple).

The treatment of pleural recurrences from thymoma is usually a matter of local control. For this reason, the report recently published by Wright ${ }^{15}$ about pleuropneumonectomy for the management of selected cases of stage IVA thymoma or pleural relapse was very provocative. The hypothesis that pleuropneumonectomy, a procedure usually reserved for malignant pleural mesothelioma, could be useful above all for thymoma is supported by the positive prognostic value of radical surgery in this slowly evolving disease. Wright treated 5 patients, 2 of whom had massive pleural recurrences from B3 thymomas. He concluded that the procedure is safe and should be part of a multimodality strategy.

Similar to the treatment of malignant pleural mesothelioma, some authors ${ }^{8,13}$ have added hyperthermic intrapleural chemotherapy to the surgical resection of pleural recurrences from thymoma. In our last 3 patients we also used hyperthermic intraoperative administration of 2 drugs (cisplatin and epidoxorubicin); the outcome is still "in progress," but the treatment is feasible with low morbidity.

Thymomas are very sensitive to radiation, ${ }^{16}$ but adjuvant radiotherapy after thymoma resection does not decrease the incidence of pleural relapses inasmuch as the implants are set out of the radiation field.

Low-dose entire hemithorax radiotherapy for pleural dissemination or relapse has been described in only a few articles, ${ }^{9,17,18}$ which showed that this method is safe and could contribute to the control of pleural dissemination.

Advanced and recurrent thymomas are also responsive to systemic chemotherapy, as reported by Giaccone and associates, ${ }^{12}$ who found a $31 \%$ response rate to chemotherapy. To decrease the toxicity of systemic chemotherapy and also to achieve better local control, Terada and colleagues ${ }^{13}$ have successfully managed 2 cases of pleural recurrences by transarterial infusion of chemotherapy through the intercostal arteries and subphrenic artery.

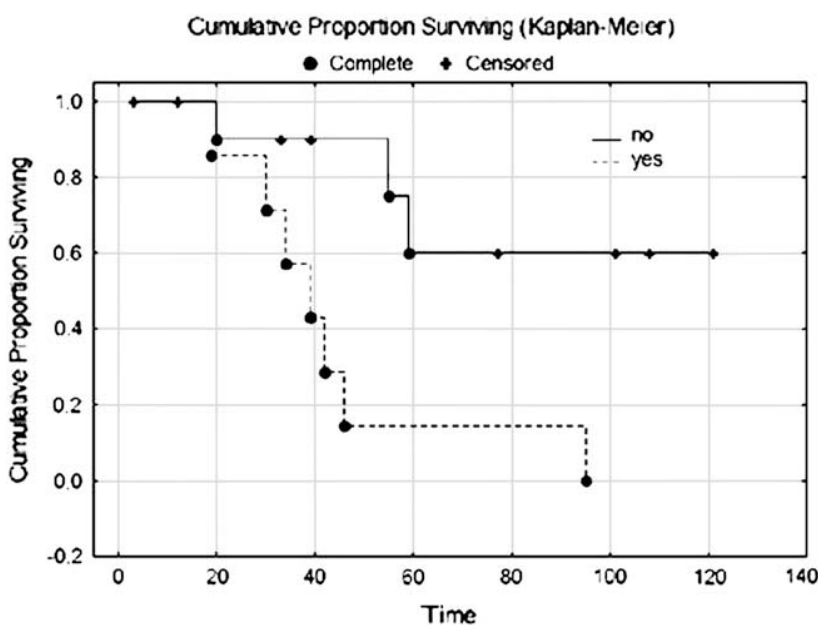

FIGURE 5. Overall survival according to the diaphragmatic involvement.

When the resection was not radical, we also decided to use adjuvant chemotherapy. Unfortunately, the literature contains no supportable evidence of its value.

The last 6 patients received additional adjuvant prednisone and octreotide according to the preliminary experience of Palmieri and coworkers ${ }^{19}$ and the Eastern Cooperative Oncology phase II trial by Loehrer and associates ${ }^{20}$ who obtained outstanding results in advanced-stage thymomas.

Even if our series are small and the period of analysis is long, we have found some interesting results. Among the analyzed variables, only diaphragmatic involvement and number of implants were statistically significant, with a worse prognosis in patients with diaphragmatic and multiple implants. A possible explanation is that multiple implants, those particularly involving the diaphragm, express a more advanced disease. This issue and the data in the literature, showing that the mean disease-free period is between 60 and 80 months, stress the role of lifetime follow-up to detect recurrences of thymoma at an early stage.

The WHO's histologic classification system reflects the oncologic behavior of thymoma, ${ }^{21-23}$ with most pleural recurrences occurring in type B3 thymoma. On the other hand, the WHO's histologic classification system is not a prognostic indicator when the survival is calculated from the occurrence of pleural relapse itself.

We avoided performing multivariable analysis, which is not meaningful with so few patients.

After surgical resection, a subsequent pleural recurrence is probable, so that a strict radiologic follow-up is mandatory in this subset of patients. When a new pleural relapse is found, an iterative resection should be considered. ${ }^{24} \mathrm{We}$ have performed 3 iterative resections of pleural implants and in 1 case a third operation was performed; all the patients were long-term survivors and 2 of them are still alive and well. 
We can conclude that reoperation for thymoma pleural recurrences is feasible and safe. The next question is an open one: Which kind of operation should be performed in the case of a pleural relapse from thymoma-simple resection of the implant, complete pleurectomy, or an extrapleural pneumonectomy? A conclusive answer is not possible and the choice is still based on the status of the disease, on the status of the patient, and on the policy of the surgeon.

The take-home message is this: Do not give up in this indolent disease. Always consider a reresection, because everything else is based on speculation and theory.

We thank Dr. Jennifer Johnson and Mrs. Paola Moretti, for their assistance in manuscript preparation.

\section{References}

1. Kondo K, Monden Y. Lymphogenous and hematogenous metastasis of thymic epithelial tumors. Ann Thorac Surg. 2003;76:1859-64.

2. Pescarmona E, Rendina EA, Venuta F, Ricci C, Baroni CD. Recurrent thymoma: evidence for histological progression. Histopathology. 1995;27:445-9.

3. Ruffini E, Mancuso M, Oliaro A, Casadio C, Cavallo A, Cianci R, et al. Recurrence of thymoma: analysis of clinicopathologic features, treatment, and outcome. J Thorac Cardiovasc Surg. 1997;113:55-63.

4. Mineo TC, Biancari F. Reoperation for recurrent thymoma: experience in seven patients and review of the literature. Ann Chir Gynaecol. 1996;85:286-91.

5. Regnard JF, Zinzindohoue F, Magdeleinat P, Guibert L, Spaggiari L, Levasseur P. Results of re-resection for recurrent thymomas. Ann Thorac Surg. 1997;64: 1593-8.

6. Haniuda M, Kondo R, Numanami H, Makiuchi A, Machida E, Amano J. Recurrence of thymoma: clinicopathological features, re-operation, and outcome. J Surg Oncol. 2001;78:183-8.

7. Ciccone AM, Rendina EA. Treatment of recurrent thymic tumors. Semin Thorac Cardiovasc Surg. 2005;17:27-31.

8. Refaely Y, Simansky DA, Paley M, Gottfried M, Yellin A. Resection and perfusion thermochemotherapy: a new approach for the treatment of thymic malignancies with pleural spread. Ann Thorac Surg. 2001;72:366-70.

9. Ichinose Y, Ohta M, Yano T, Yokoyama H, Asoh H, Hata K. Treatment of invasive thymoma with pleural dissemination. J Surg Oncol. 1993;54:180-3.
10. Kataoka D, Nonaka M, Yamamoto S, Fukuzumi M, Kunimura T, Kaga E, et al Experience with invasive thymoma presenting pleural dissemination. Kyobu Geka. 2003;56:1025-8.

11. Taguchi T, Suehiro T, Toru K, Ogami N, Takata H, Hashimoto K. Pleural dissemination of thymoma showing tumor regression after combined corticosteroid and tacrolimus therapy. Eur J Intern Med. 2006;17:575-7.

12. Giaccone G, Wilmink H, Paul MA, van der Valk P. Systemic treatment of malignant thymoma: a decade experience at a single institution. Am J Clin Oncol. 2006; 29:336-44.

13. Terada Y, Kambayashi T, Okahashi S, Noguchi T, Kamakari K, Kubo S. Transarterial infusion chemotherapy for recurrence of pleural dissemination after thymectomy. Ann Thorac Surg. 2005;79:e32-3.

14. De Bree E, van Ruth S, Baas P, Rutgers EJ, van Zandwijk N, Witkamp AJ, et al Cytoreductive surgery and intraoperative hyperthermic intrathoracic chemotherapy in patients with malignant pleural mesothelioma or pleural metastases of thymoma. Chest. 2002;121:480-7.

15. Wright CD. Pleuropneumonectomy for the treatment of Masaoka stage IVA thymoma. Ann Thorac Surg. 2006;82:1234-9.

16. Curran WJ Jr, Kornstein MJ. Brooks JJ, Turrisi AT 3rd. Invasive thymoma: the role of mediastinal irradiation following complete or incomplete surgical resection. J Clin Oncol. 1988;6:1722-7.

17. Yoshida H, Uematsu M, Itami J, Kondo M, Ito H, Kubo A, et al. The role of lowdose hemithoracic radiotherapy for thoracic dissemination of thymoma. Radiat Med. 1997;15:399-403.

18. Sugie C, Shibamoto Y, Ikeya-Hashizume C, Ogino H, Ayakawa S, Tomita N, et al. Invasive thymoma, postoperative mediastinal irradiation, and low-dose entire hemithorax irradiation in patients with pleural dissemination. J Thorac Oncol. 2008;3:75-81.

19. Palmieri G, Lastoria S, Colao A, Vergara E, Varrella P, Biondi E, et al. Successful treatment of a patient with a thymoma and pure red-cell aplasia with octreotide and prednisone. N Engl J Med. 1997;336:263-5.

20. Loehrer PJ Sr, Wang W, Jonson DH, Ettinger DS. Eastern Cooperative Oncology Phase II Trial. Octreotide alone or with prednisone in patients with advanced thymoma and thymic carcinoma: an Eastern Cooperative Oncology Group Phase II Trial. J Clin. Oncol. 2004;22:293-9.

21. Okumura M, Ohta M, Tateyama H, Nakagawa K, Matsumura A, Maeda H, et al. The World Health Organization histologic classification system reflects the oncologic behavior of thymoma. Cancer. 2002;94:624-32.

22. Kondo K, Yoshizawa K, Tsuyuguchi M, Kimura S, Sumitomo M, Morita J, et al WHO histologic classification is a prognostic indicator in thymoma. Ann Thorac Surg. 2004;77:1183-8.

23. Lucchi M, Basolo F, Ribechini A, Ambrogi MC, Bencivelli S, Fontanini G, et al Thymomas: clinical-pathological correlations. J Cardiovasc Surg. 2006;47:89-93.

24. Sakada T, Sugio K, Nishioka K, Tsukamoto S, Ushijima C, Yamazaki K, et al Invasive thymoma with long-term survival by extensive reoperation. Respiration. 1999;66:167-9. 